NHS management structure improved from 33\% to $93 \%$. $100 \%$ of the delegates who attended the course found it helpful and would recommend it to a colleague. $90 \%$ of the delegates would consider taking on more leadership and management roles in the future. These findings suggest that there is a great need for such courses to narrow the leap from being a registrar to a consultant.

\section{TRAINEES TAKING CHARGE - DESIGN AND DELIVERY OF A PACES PREPARATION COURSE}

${ }^{1}$ Nora van der Stelt, ${ }^{2}$ Amy Coulden, ${ }^{3}$ Imogen Sutherland, ${ }^{4}$ Anna Naito. ${ }^{1}$ Ealing Hospital, Southall, Middlesex, UK; ${ }^{2}$ Queen Elizabeth Hospital, Birmingham, UK; ${ }^{3}$ Frimley Park Hospital, Camberley, UK; ${ }^{4}$ King's College Hospital, London, UK

\subsection{6/leader-2021-FMLM.73}

Membership of the Royal College of Physicians (MRCP) is compulsory for Internal Medical Trainees (IMTs) moving into higher specialty training. The practical component of MRCP the PACES (Practical Assessment of Clinical Examination Skills) exam - requires extensive preparation. No structured PACES teaching programme existed at St Helier Hospital, UK. In the authors' experience, local PACES preparation courses are often organised by those who themselves sat the exam many years ago, whereas trainees with recent exam experience are in an excellent position when it comes to providing preparation support.

The authors, at the time all IMTs with recent PACES experience, designed and delivered a teaching programme over a three-month period. The course consisted of weekly bedside and simulated teaching sessions tailored to candidates' specific needs, and a pan-London mock PACES exam to give candidates the opportunity to practise their skills in an environment closely resembling the real-life experience.

Direct feedback was obtained both pre and post course, and following each individual session. Questions focussed on such issues as ease of access to PACES teaching and perceived exam preparedness. Pre-course feedback indicated that $86 \%$ of candidates felt teaching was difficult to obtain, whereas post course $100 \%$ felt this was now easily available. Perceived preparedness across all stations increased from 2.3-3.9 on a 10point scale pre-course, to $7.8-8.7 / 10$ post-course.

The success of this project stressed the importance of realising that leadership is not so much a matter of seniority as it is of experience. Having only recently sat the exam themselves, the authors were in a unique position to identify the needs of PACES candidates and lead a successful preparation programme. Not only is this beneficial for exam candidates; it offers junior trainees the opportunity to develop and display leadership skills that will prove invaluable throughout the rest of their careers.

\section{USE OF SIMULATION TO DEVELOP LEADERSHIP}

Daniel Campbell. South Tyneside and Sunderland NHS Foundation Trust, UK

\subsection{6/leader-2021-FMLM.74}

Aims To investigate the impact of participation in acute simulation sessions on foundation doctors perceived confidence in their leadership abilities.
Methods Data was collected from participants who attended a foundation programme acute simulation session. They were asked to complete a pre and post session questionnaire regarding their perceived confidence in their leadership abilities, which they rated using a 5-point Likert scale. The pre and post score averages were calculated to ascertain if there was a change in the participants perceptions following the session.

Results 60 participants attended the session and completed the questionnaire. When asked about their confidence in leading whilst managing acutely unwell patients the pre-session average was 2.7 and post average score 3.8. When asked about completing an $\mathrm{ABCDE}$ assessment and making an initial management plan the pre-session average was 3.8 and post-session 4.8 , and when asked about their confidence in recognising the limitations of their abilities the pre-session average was 4.2 and the post-session average 4.6.

Conclusions The results of this project have highlighted the low confidence foundation doctors perceived in their abilities to lead in acute situations. Conversely, they felt confident in recognising situations where they had reached the limitation of their abilities. There was a positive outcome from attending the session with an increase of 1.1 in the average score concerning participants confidence in leading acute situations and 1.0 in performing an ABCDE assessment and making an initial management plan. Overall, this work has demonstrated that attendance at an acute simulation session has improved the foundation doctor's confidence in their leadership abilities. Further work is needed to ascertain what factors influence junior doctor's perceptions of their leadership skills and whether the reported improvement in confidence impacted their clinical practice.

\section{USING A BLENDED LEARNING APPROACH TO DEVELOP QUALITY IMPROVEMENT SKILLS AMONGST JUNIOR DOCTORS}

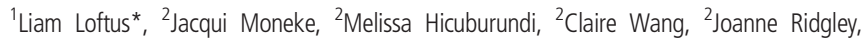
${ }^{2}$ Rebecca Bennewith, ${ }^{2}$ Natasha Aghtarafi. ' GP Trainee, Cambridge, and Health Education East of England Leadership Fellow; ${ }^{2}$ Foundation Year Doctor, Cambridge University Hospital

\subsection{6/leader-2021-FMLM.75}

Aims Leadership competencies and quality improvement skills are becoming an increasingly important element of junior doctor training curricula. How we support our junior doctors to develop these skills must therefore advance to match this.

This work aimed to take a blended learning approach, using eLearning modules alongside a real-world project, to develop quality improvement (QI) capability amongst Foundation Year (FY) doctors.

Methods Four eLearning modules were created by Health Education East of England Leadership Fellows, which covered all the core components of completing a quality improvement project (QIP). A Leadership Fellow then engaged six FY doctors, who collaborated on four QIPs, which aimed to increase the number of Fit Notes issued to patients upon discharge from a large teaching hospital.

They received direction from the eLearning modules, in addition to light-touch supervision from a Leadership Fellow (specifically an initial meeting, 4-6 weekly catch-up emails, and ad-hoc troubleshooting). 
Results $100 \%$ of the FY participants who responded to the post-project evaluation survey $(n=4)$ either agreed, or strongly agreed, with the following:

- I now have a greater understanding of how to complete a QIP.

- The amount of supervision I received was appropriate.

- I would recommend this model of learning to a colleague.

Each QIP demonstrated a positive impact on patient care, and included all of the core elements necessary to meet QI curriculum requirements.

Conclusion This work demonstrates how a blended approach can drive the development of QI skills to the point of expected competency, while also delivering successful QIPs that result in improved quality of patient care.

\section{USING FRAILTY REVIEWS TO MANAGE THE COVID-19 PANDEMIC IN AN EVIDENCE-BASED AND PERSON- CENTRED MANNER ACROSS BUCKINGHAMSHIRE CARE HOMES}

${ }^{1}$ Unoma Okoli* ${ }^{1}$ Shahina Juma ${ }^{1}$ Sheena Patel, ${ }^{1}$ Monice Hussain ${ }^{2}$ Ana Phelps, ${ }^{2}$ Beatrix Nagyova, ${ }^{2}$ Theresa Frang, ${ }^{2}$ Chi Yuen Yau, ${ }^{2}$ Veena Borkar, ${ }^{2}$ Stephanie Tanner, ${ }^{2}$ Jo Birrell. ${ }^{1}$ Buckinghamshire Clinical Commissioning Group, UK; ${ }^{2}$ Buckinghamshire Healthcare NHS Trust, UK

\subsection{6/leader-2021-FMLM.76}

Aim NICE COVID-19 guidelines NG191 recognise that frailer patients, including those with a higher Rockwood Clinical Frailty Scale ${ }^{1}$ (CFS), are seldom improved by hospital admission.

The project used a collaborative approach to undertake frailty reviews, aiming to reduce inappropriate hospital admissions. The reviews included discussing residents' preferred place of care and options in the event of deterioration, a Treatment Escalation Plan (TEP).

Method A collaborative was formed between care home managers, pharmacists and geriatricians to review frailty factors in residents over 65 years, excluding those with Learning Disabilities. Care homes with high risk of COVID-19 were prioritised. A Standard Operational Procedure was approved within one month. Using existing resources only, the project was delivered over 3 months. Communication was sent to GP practices, care homes and residents/families and consent was obtained. Templates were developed and education delivered.

Results Review outcomes were recorded electronically onto the GP clinical system. Care homes kept a copy and informed residents/families of the outcome. 595 residents were reviewed in 21 care homes.

Preliminary analysis for 71 residents reviewed May 2020 to April 2021 show:

- 42 survivors, 33 with severe frailty (CFS 7-9), 9 not severely frail $(\mathrm{CFS}<7)$. Out of total 28 residents with TEP indicating avoidance of hospital admission, 23 were not admitted and 5 had single hospital admission. 14 residents TEP included hospital management of whom 7 were admitted.

- 26 of the 29 deceased residents were severely frail. 96\% died in their preferred place of death.

Conclusion Clinical leadership demonstrated courage and capacity to challenge the status quo, improve organisational processes and innovate practice. This helped staff morale at a difficult time of immense pressure and positively enhanced care homes' profile and residents' experience.

\section{REFERENCE}

1. K Rockwood, et al. CMAJ 2005;173:489-495.

\section{USING PDSA (PLAN - DO - STUDY - ACT) MODEL TO INCREASE MEDICINE RECONCILIATION IN A TERTIARY CARE HOSPITAL OF A DEVELOPING COUNTRY}

Amber Sabeen, Abdul Aziz, Ambreen Amirali. Aga Khan University Hospital, Karachi Pakistan

\subsection{6/leader-2021-FMLM.77}

Objective Using PDSA (Plan - Do - Study - Act) model to increase medicine reconciliation in a tertiary care hospital of a developing country.

Background The WHO defines the medication reconciliation process as the formal process in which health care professionals partner with patients to ensure precise and complete medication information transfer at different interfaces of care'. Medication errors at time of a patient's hospital admission are common occurrence in health care settings. Majority of the error occurs during prescribing, dispensing or administration of medicines. In order to avoid these errors, medication reconciliation processes and systems have been employed by health care organizations throughout the world. Implementing medical reconciliation has successfully reduced medication error in many institutes.

Study Design and Methodology It was a quality improvement project conducted in the Department of Medicine, Aga Khan University Hospital Karachi. We included residents and interns working in Medicine department. The written proforma was distributed in three sessions for three consecutive weeks. The PDSA model was implemented for four months from February 2019 to May 2019 in the department of internal medicine.

Result The Medicine reconciliation compliance improved from 4\% in February 2019 to $96 \%$ in May 2019.

Conclusion With the help of PDSA cycle we advised and managed to implement quality improvement interventions and changes that resulted in significant improvement in medication reconciliation compliance. This strategy of PDSA cycle can be applied in other quality indicator projects also for increasing patient safety and decrease preventable harm. This project also shows that engaging the health care workers will overcome the resistance to change and implement sustainable systems.

\section{WHAT KIND OF PERCEPTIONS DO THE MEDICAL AND INFORMATION DIRECTORS HAVE ABOUT THE FACTORS INFLUENCING ON KNOWLEDGE MANAGEMENT IN HOSPITAL?}

Milla Kajanne. Researcher, University of Helsinki, Programme in Social and Health Research and Management, Finland

\subsection{6/leader-2021-FMLM.78}

Aims The aim of the study is to explore, how the systematic knowledge-based management and the assessment of effectiveness have been implemented in hospitals in Finland. What are the potential bottlenecks and practical challenges on the way 